\title{
Reflets
}

Revue ontaroise d'intervention sociale et communautaire

\section{Intervention et crise suicidaire}

\section{Cilvy Dupras}

Volume 6, numéro 1, printemps 2000

Approches d’intervention : définir et renouveler nos pratiques

URI : https://id.erudit.org/iderudit/026303ar

DOI : https://doi.org/10.7202/026303ar

Aller au sommaire du numéro

Éditeur(s)

Reflets : Revue ontaroise d'intervention sociale et communautaire

ISSN

1203-4576 (imprimé)

1712-8498 (numérique)

Découvrir la revue

Citer cet article

Dupras, C. (2000). Intervention et crise suicidaire. Reflets, 6(1), 197-207.

https://doi.org/10.7202/026303ar

Tous droits réservés (C) Reflets : Revue ontaroise d'intervention sociale et communautaire, 2000

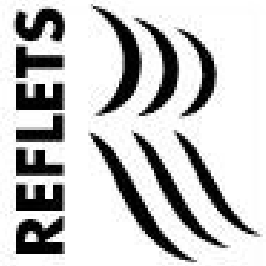

Ce document est protégé par la loi sur le droit d'auteur. L'utilisation des services d'Érudit (y compris la reproduction) est assujettie à sa politique d'utilisation que vous pouvez consulter en ligne.

https://apropos.erudit.org/fr/usagers/politique-dutilisation/ 


\title{
Intervention et crise suicidaire
}

\author{
Cilvy Dupras,
}

M.A., Sudbury

Dans cet article, j'aimerais illustrer quelques principes d'intervention en situation de crise. Pour ce faire, j'utiliserai quelques mises en situation pouvant survenir, dans divers milieux de pratiques, lors de crise suicidaire. Ces principes pourraient vous aider à reconnaitre quelques-uns des signes annonciateurs émis par une personne voulant se suicider et, éventuellement, lui venir en aide. Ainsi, plus il y aura de gens qui savent reconnaitre les risques et intervenir dans de telles situations, plus on réussira à réduire le nombre de gestes suicidaires. En ce sens, ce court article tente d'inciter toute personne voulant aider une personne qui songe à s'enlever la vie, à rechercher une formation professionnelle adéquate pour intervenir efficacement.

\section{Une situation}

Il est quatre heures du matin. Vous êtes bénévole à une ligne de crise. Le téléphone sonne, vous répondez. Une personne à la voix éteinte, comme celle d'une dame âgée, vous demande de vos nouvelles. Après lui avoir dit que vous allez bien, vous lui demandez comment vous pouvez l'aider. Elle ne répond pas à votre question. Elle préfere continuer à vous interroger sur ce que vous avez mangé au souper ou sur la dernière visite que vous avez rendue à votre mère. Elle veut savoir si vous conduisez de façon imprudente à l'occasion. Elle insiste sur votre relation avec votre mère. Vous répondez brièvement et tentez de ramener la dame à la raison de 
son appel. Elle continue de discuter et revient souvent sur la relation avec votre mère. La conversation dure ainsi une bonne demi-heure et la gentille dame raccroche sans rien livrer d'ellemême.

Quelques jours plus tard, vous lisez dans le journal local qu'une femme âgée est morte, la nuit même où vous avez reçu cet appel. L'article mentionne que peu de temps auparavant, elle venait de perdre son fils unique dans un accident de voiture. Était-ce la même dame? S'est-elle enlevée la vie? Vous n'en savez rien. Mais vous vous demandez si vous auriez pu aider davantage la femme qui vous a téléphoné? Si non, pourquoi? Si oui, comment? Où trouve-t-on ces réponses?

\section{Le suicide}

On définit le suicide comme un acte délibéré, un comportement autodestructeur entraînant la mort. C'est là une définition très factuelle. C'est sur cette base qu'on détermine l'incidence du suicide dans la population ontarienne. Ce faisant, on se bute à des données incomplètes puisqu'elles ne présentent que les cas de suicide complétés et dont l'évidence ne peut être mise en doute ${ }^{1}$. Ainsi, selon les données sur les causes connues de mortalité, on estime que $12 \%$ de femmes et $18 \%$ d'hommes sont décédés suite à un acte suicidaire (PHRED Partners 2000). À titre indicatif du taux de suicide, Ramsey et al. (1997) indiquent qu'au Canada, celui-ci est aujourd'hui de 14 personnes pour 100000 habitants, un taux à peu près identique dans tous les groupes socioéconomiques. Ils estiment aussi que 700 jeunes se donnent la mort tous les ans, ce qui équivaut à près de deux décès par jour. Ils ajoutent que le taux de suicide augmente avec l'âge chez les deux sexes et qu'en Amérique du Nord, le taux de suicide des autochtones est environ trois à quatre fois plus élevé que celui de l'ensemble de la population. Le suicide affecte aussi certains groupes de la population dont les groupes ethniques, les homosexuels et les personnes atteintes de maladie mentale. 
Pourtant, ces chiffres et cette définition factuelle du suicide ne tiennent pas compte de la souffrance de la personne. En effet, selon Séguin et Huon, la personne suicidaire est une personne qui souffre. «C'est une personne qui vit une crise dont l'ampleur la dépasse, elle se sent impuissante et n'arrive pas à diminuer la souffrance et le désarroi qu'elle vit» (1999: 16). Au niveau de l'intervention, cela souligne l'importance déterminante de percevoir et d'écouter cette souffrance.

Nous ne pouvons plus, à l'heure actuelle, être indifférents par rapport au suicide. Au contraire, il faut intervenir et utiliser tous les moyens mis à notre disposition pour prévenir ce phénomène. Voilà pourquoi, ma collègue Colette Mann de l'Association de la santé mentale et moi-même avons choisi d'offrir des ateliers d'intervention sur le suicide, à l'invitation de certains membres et de groupes de notre communauté qui voulaient que ça change ${ }^{2}$.

Certes, plusieurs méthodes d'intervention existent, mais celle que nous privilégions est de permettre aux personnes qui assistent aux ateliers $\mathrm{C}$ ateliers que nous offrons en français $\mathrm{C}$ de pratiquer l'intervention à partir de mises en situation plausible de crise suicidaire. Il s'agit d'ateliers qui offrent l'occasion aux participants et aux participantes d'appliquer les techniques et les principes d'intervention. En effet, selon nous, l'apprentissage se fonde sur l'expérience, les discussions, les échanges, l'expression des sentiments et la confrontation des attitudes liées au suicide.

Les personnes qui assistent aux ateliers proviennent de tous les groupes d'âges, de tous les milieux et de toutes les professions. Plusieurs reviennent faire la formation (quatre ou cinq fois) afin de renforcer ce qu'ils ont appris et pour se sentir vraiment à l'aise à intervenir.

\section{Quelques prémisses de l'intervention}

Au début de l'article, je présentais la situation d'une dame âgée. Or, toute travailleuse ou tout travailleur œuvrant à une ligne de 
crise, sait que ce genre d'appel est possible. Toutefois, on ne peut intervenir que si les personnes manifestent leur intention suicidaire de façon claire. En effet, dans la majorité des cas, les personnes donnent des signes annonciateurs de la façon dont ils réagissent aux événements et qui les poussent à passer à l'acte suicidaire (Ramsey et al. 1997).

Pourtant, on sait aussi que certaines personnes peuvent se suicider sans rien annoncer. Par conséquent, même avec les meilleures intentions et la meilleure formation qui soit, il n'est pas possible d'aider ou de soutenir tout le monde. Par contre, l'intervention de crise, présentée dans cet article, permet de faire la lumière sur le monde de l'intervention d'urgence en cas de suicide. J'aimerais aussi souligner qu'en intervention de crise, rien ne vaut la pratique, c'est-à-dire la mise en situation plausible afin de développer des habiletés et de se sentir à l'aise. Ainsi, dans la formation que nous offrons, Colette et moi faisons appel à certaines de nos expériences, à celles de nos collègues et à celles de personnes de tous les milieux qui suivent nos ateliers de formation sur le suicide.

Les intervenantes et les intervenants de crise peuvent confirmer que les premières personnes qui interviennent en cas de crise suicidaire sont rarement les thérapeutes. Et ma pratique illustre cet état de fait, puisque j'ai rarement rencontré des personnes qui venaient me consulter à cause d'une crise suicidaire. Par contre, j'ai rencontré des personnes suicidaires dans ma famille, dans mon travail en maison de transition et dans les couloirs des hôpitaux. J'en ai aussi croisé dans mon équipe de natation et dans mes cours au collège et à l'université. J'ai rencontré des survivantes et des survivants de personnes qui avaient vécu une crise suicidaire, mortelle ou non. Ces personnes ont fréquemment besoin d'aide. Mais comment pouvons-nous les aider?

Il faut se demander si une personne doit avoir des caractéristiques particulières pour intervenir en cas de crise suicidaire. D'abord, il est important de rester calme dans des situations de crise. Murray Firth, dans le cours "Critical Incident Stress Management», dit que les personnes qui choisissent l'intervention de crise (ou d'urgence) comme profession, ont des traits de personnalité assez particuliers. Toutefois, la majorité des 
personnes qui interviennent ne sont pas des professionnelles ou des professionnels. Ainsi, on ne doit pas se transformer en sauveur, chercher à être une héroïne ou un héros. Il s'agit avant tout de savoir quoi faire en pareilles circonstances et de l'avoir déjà pratiqué. D'où l'importance pour toute personne de suivre une formation qui lui permettra de développer certaines habiletés et d'apprendre certaines techniques pour intervenir.

\section{Une deuxième situation}

Vous êtes intervenante en maison de transition. Vous rencontrez une femme qui a déjà été vendeuse de drogue et qui a aussi fait de la prostitution. Elle est séropositive et a transmis le virus à son enfant. Elle vient vous voir au milieu de la nuit et vous dit qu'elle ne veut pas voir le soleil se lever. Quel est votre premier réflexe?

La majorité d'entre nous fera comme si elle n'avait rien entendu ou banalisera la portée de ces paroles en disant: «c'est «normal». Ce premier réflexe passé, nous pouvons et devons chercher à en connaître davantage. Qu'est-ce qui se passe au juste? Ai-je bien entendu? S'agit-il vraiment de ce que je pense? Selon Séguin (1991) et Ramsay et al. (1997), il n'y a rien de mieux à faire dans ces circonstances que d'indiquer à la personne notre disponibilité, de lui dire que nous sommes là pour l'écouter. Colette Mann dit qu'après être engagé dans une situation de crise, nous faisons tout notre possible (de façon réaliste) pour «aller jusqu'au bout de l'intervention avec la personne». Il faut choisir rapidement de s'impliquer ou non.

Il peut y avoir des raisons très valables pour décider de ne pas s'embarquer dans un processus d'intervention. Dans cette éventualité, il est important de pouvoir offrir une série de ressources disponibles et de s'assurer que ces ressources interviennent.

S'engager est la première étape d'une intervention en situation de crise. On choisit de foncer et de vivre avec les conséquences, 
notamment celle de rester auprès de la personne aussi longtemps que cela est nécessaire. Nous savons qu'avec de l'aide, les crises suicidaires aiguës se résorbent généralement en quelques heures, mais la personne suicidaire peut l'ignorer parce qu'elle vit avec intensité, sa douleur et son désespoir depuis trop longtemps, ce qui l'empêche de trouver des solutions.

Ensuite, on l'écoute. On pose des questions sur sa situation. On demande des précisions. On insiste sur les détails. Il faut parfois demander directement «Es-tu en train de me dire que tu veux en finir avec ta vie?» ou «Es-tu suicidaire?». La personne pourrait alors se sentir soulagée de voir que nous n'avons pas peur des mots et des émotions difficiles. Elle pourrait nous le confirmer ou au contraire, nous dire que ce n'est pas ce dont il s'agit et passer à autre chose.

\section{Une troisième situation}

Vous discutez avec la meilleure amie de votre adolescente qui profite de ce face-à-face pour vous dire qu'elle veut mourir. Vous apprenez que votre fille était au courant mais qu'elle avait promis de ne pas en parler avant que sa meilleure amie ne le fasse ellemême. Vous êtes estomaquée et affolée. Vous avez peur pour votre propre fille. Du même souffle, vous réalisez que vous connaissez les parents de l'adolescente et vous vous demandez comment ils réagiraient? Les questions qui vous trottent dans la tête sont: «Comment une si belle adolescente peut avoir des idées comme ça? Tout semble lui réussir, comment peut-elle en être arrivée là?». Dans une telle situation, il s'agit de lui demander, tout simplement. Si vous écoutez avec une attitude suffisamment ouverte et respectueuse, elle vous répondra probablement. Elle ne vous donnera pas toutes les réponses. Par contre, avec de la patience et de la persévérance, vous obtiendrez assez d'informations pour vous faire une idée sur sa douleur et son désespoir. Et vous pourrez probablement déterminer ainsi la partie d'elle qui veut en finir avec la vie et celle qui veut demeurer en vie. 
Mais, attention, il ne faut en aucune circonstance imposer vos propres raisons ou vos propres solutions à l'autre, ça ne fonctionnerait tout simplement pas. Il s'agit d'être vraiment le plus à l'écoute possible afin de permettre à la personne de trouver ses raisons de vivre et ses solutions au problème. Ses raisons de vivre pourraient vous sembler banales. Par exemple, elle pourrait vouloir assister au prochain concert rock ou exprimer une autre raison d'apparence anodine à vos yeux, mais qui, pour elle, lui permettra de se raccrocher à la vie pour l'instant. Ses raisons peuvent aussi être plus profondes, de l'ordre d'une quête spirituelle et de sens ou d'un lien avec une personne aimée, etc. À l'opposé, la personne peut vous donner autant de raisons de mourir. Par exemple, ses parents ont toujours fondé de grands espoirs en elle et elle ne veut plus les décevoir ou encore que la religion nous promet une meilleure vie de l'autre côté, etc. Il n'y a pas de bonnes ou de mauvaises raisons. Il n'y a que les motivations humaines à vivre ou à mourir. Et personne ne peut les juger, il faut les accepter avec le plus grand respect possible.

\section{Explorer le risque}

L'important, dans l'intervention d'urgence, est d'explorer, autant que possible, les deux côtés de la médaille pour aider la personne qui songe au suicide à découvrir une lueur d'espoir, si mince soit-elle. Et c'est à ce moment-là que nous pouvons en arriver à évaluer le risque de passage à l'acte, dans un futur plus ou moins immédiat. En fait, ce que nous devons évaluer, c'est le niveau d'ambivalence de la personne face au suicide. Si elle est très ambivalente, elle n'aura probablement pas de plan défini et n'envisagera pas le suicide comme la seule solution acceptable à ses yeux. À cet égard, rappelons qu'une personne qui a déjà tenté de se suicider ou qui connaît une personne significative qui l'a fait, a 40 fois plus de chance de trouver l'idée acceptable qu'une personne sans passé suicidaire (Ramsay et al. 1997). Si la personne est ambivalente face au suicide, elle épuisera probablement les 
quelques ressources encore à sa disposition et les explorera avant de mettre à exécution ses projets morbides. Finalement, si la personne n'est pas ambivalente, qu'elle présente un risque élevé et que ses intentions sont imminentes, l'intervention doit être immédiate et, souvent, l'on doit ou l'on peut choisir de prendre contact avec des services d'urgence, de mobiliser un réseau de soutien, d'engager les ressources professionnelles disponibles et de faire un suivi actif et intense auprès de la personne suicidaire (Séguin et Huon 1999). C'est donc à partir d'une bonne évaluation du risque et de la situation que nous pouvons aider la personne à traverser sa crise suicidaire. Ainsi, elle pourra nous aider à mettre un plan d'action en marche qui sera probablement suffisamment proche de sa réalité, et donc, qui a des chances de réussir.

\section{Établir une entente}

Vous êtes intervenante dans un centre d'intervention d'urgence. Vous accueillez une femme récemment mariée. Après l'avoir écouté attentivement, elle vous raconte qu'elle a beaucoup de difficultés dans sa vie sexuelle. Elle dit se sentir très coupable et éprouver une très grande honte lorsqu'elle fait l'amour. Maintenant qu'elle est mariée, ces sentiments sont encore plus présents et nuisent à la qualité de ses relations sexuelles. Plus jeune, elle a eu des relations sexuelles avec un membre de sa famille et elle ne pouvait pas les refuser. Elle est soulagée enfin de pouvoir le dire. Par contre, son mariage a exacerbé son malaise et a ajouté un stress qu'elle trouve intolérable. Elle ne veut pas continuer comme ça et faire subir à son conjoint, ses problèmes pour le restant de ses jours. Elle dit qu'elle a déjà pensé à prendre tous ses médicaments. Elle ajoute qu'elle considère que la meilleure solution est d'en finir et ainsi de libérer son mari des liens qui les unissent. Elle mentionne pourtant qu'elle l'aime profondément et qu'elle a toujours souhaité fonder une famille avec lui.

Vous avez beaucoup d'informations sur la situation, mais elle semble sans issue. La femme semble bien décidée à mettre son 
plan de suicide en action. Elle a pourtant offert un indice, un point sur lequel vous devez vous accrocher, celui de l'amour pour son mari et son souhait de fonder une famille. C'est probablement la seule piste que vous avez actuellement pour faire un contrat avec elle. Suivez-la le plus loin possible.Votre demande doit être précise. Par exemple, après avoir compris ses sentiments négatifs et ses aspects positifs, tentez d'envisager avec elles d'autres pistes de solution qui pourraient l'aider à vivre. À mesure que les solutions de rechange se présenteront, la personne peut envisager sa situation avec une nouvelle perspective. Demandez-lui de ne pas se faire mal jusqu'à votre prochaine rencontre de demain midi ou pour la prochaine journée. Demandez-lui de faire une liste des aspects positifs et négatifs de sa situation et de se donner une chance de plus, etc. Il s'agit de développer des objectifs précis et réalistes pour que la personne puisse tenir un engagement et le réussir. Pour ce faire, demandez-lui de vous répéter l'engagement que vous venez de conclure avec elle et assurez-vous qu'elle consentira à le respecter. Finalement, aidez-la à mobiliser son réseau de soutien et à prévoir une forme d'aide en cas d'urgence et, assurez-vous que cette aide sera disponible en temps voulu.

En dernier lieu, et non le moindre, faites un retour sur l'intervention le plus rapidement possible avec une ou un collègue ou avec votre superviseure ou votre superviseur. En effet, rappelezvous que c'est vous qui venez d'absorber le trop plein d'émotions et il est important de le diffuser. Nous n'avons pas besoin de répéter les conséquences néfastes de passer outre ses émotions ou encore, celles d'un burnout.

\section{Conclusion}

Aider des personnes suicidaires est une tâche exigeante et troublante. Même lorsqu'on a fait des études dans les domaines du service social ou de la santé, on manque souvent d'expérience pratique à ce niveau. Si vous avez à aider une personne suicidaire, n'hésitez pas à le faire. En ce sens, l'Association canadienne pour 
la santé mentale recommande les points suivants comme trousse de premiers soins pour prévenir les comportements suicidaires.

- Engagez la relation et amenez la personne à parler de ses sentiments sur les événements qui la perturbent.

- Observez bien les signes indiquant des intentions suicidaires, croyez-en vos intuitions et votre jugement.

- Vérifiez auprès de la personne concernée pour les signes moins évidents.

- Montrez-lui votre intérêt et écoutez ce qu'elle a à dire.

- Demandez-lui si elle a des intentions suicidaires.

- Montrez-lui autant de respect et de compréhension que possible. Soyez honnête et sincère et prenez la situation au sérieux.

- Impliquez-vous activement, dès que c'est possible. Parlez ouvertement et librement et posez des questions directes.

- Déterminez le niveau de risque actuel. Demandez s'il y a eu des idées suicidaires ou s'il a déjà fait une ou des tentatives suicidaires. Informez-vous pour savoir si la personne a un plan d'action.

- Offrez de l'aide et du soutien, laissez la personne parler. Concentrez votre attention pour faire quelque chose à propos des idées et des comportements suicidaires. Offrez de l'espoir et des ressources réalistes pour la personne.

- Demandez de l'aide, pour vous et pour la personne que vous aidez. Évitez les pactes secrets. Demandez à la personne de vous dire explicitement comment elle s'y prendra pour avoir réellement de l'aide significative pour elle. Aidez la personne à rejoindre cette aide si nécessaire

- Concluez une entente pour réduire les risques.

- Donnez suite à vos engagements.

- Soyez précis dans les mesures à prendre et ayez des objectifs restreints.

- Faites un suivi auprès de la personne (Ramsey et al. 1977, traduction libre).

Ce ne sont là que quelques conseils et techniques pouvant faciliter la relation avec une personne suicidaire. Il n'y a pas de formule magique pour aider quelqu'un qui risque de se suicider.Toutefois, 
une formation solide peut vous permettre d'intervenir en situation de crise suicidaire et de réduire le risque, voire d'empêcher un suicide.

\section{Bibliographie}

BER GERON,André et ÉricVOLANT (1998). Le suicide et le deuil, Montréal,Éditions du Méridien, Collection santé.

CARREFOUR INTERVENTION SUICIDE (1999). Comment aider, Sherbrooke, Carrefour intervention suicide.

RAMSAY, R.F., B.L.TANNEY, R.J.TIERNEY et W.A. LANG (1997). Suicide : Guide d'intervention, Calgary, LivingWorks Education Inc.

SÉGUIN, Monique (1991). Le suicide. Comment prévenir, comment intervenir, Montréal, Éditions Logiques. SÉGUIN, Monique et Phillippe HUON (1999). Le suicide. Comment prévenir, comment intervenir, Montréal, Éditions Logiques.

Note

1. Le verdict de suicide est fondé sur des preuves précises. Il est donc sous-déclaré par manque de preuves, celles-ci pouvant être cachées, négligées ou pour éviter la stigmatisation sociale. Par exemple, certains accidents de la route, le décès de piétons, des empoisonnements, etc., peuvent être considérés comme des accidents et non des suicides (Ramsey et al. 1997; PHRED Partners 2000).

2. Les ateliers sont bâtis à partir des travaux de Ramsey et al. (1997). Pour recevoir la formation en français dans le Nord de l'Ontario, contacter Cilvy Dupras à Options communications au (705) 669-1200 ou Colette Mann à l'Association canadienne pour la santé mentale bureau de Sudbury au (705) 675-5272. Pour devenir formatrice ou formateur ou pour recevoir de l'information, contactez Living Works à Calgary au (403) 209-0242. 